\title{
Design of Signal Transceiver System Based on High Temperature Sensor SiCN
}

\author{
Tianli Li, Donghui Zhou, Gang Xu* ${ }^{*}$ Yuansi Chen and Junli Xu \\ Shenzhen Key Laboratory of Electromagnetic Control, Shenzhen University, Shenzhen 518060, China \\ ${ }^{*}$ Corresponding author
}

\begin{abstract}
This paper puts forward the passive and wireless SiCN temperature sensor in the applicability and necessity of the turbine engine internal environment temperature measurement. Using the principle of resonance frequency scanning tracking method based on perturbation to be the system test method, while the sensor feedback signal and the input signal ratio $\left(S_{11}\right)$ parameter as data analysis, and establish the relationship between the resonance frequency and temperature by means of tests. Experimental results show that with increasing temperature from $25^{\circ} \mathrm{C}$ to $300^{\circ} \mathrm{C}$, the $\mathrm{SiCN}$ sensor resonance frequency decreases. Corresponds to the theoretical derivation, and the minimum frequency temperature gradient is $45.92 \mathrm{kHz} /{ }^{\circ} \mathrm{C}$. Therefore, under certain conditions, the hardware system can replace the network analyzer to work at the temperature test of SiCN sensor.
\end{abstract}

Keywords-SiCN; temperature sensor; sweeping microwave; resonance frequency

\section{INTRODUCTION}

It is essential to observe the temperature and pressure continuously to get greater efficiency and less pollution requirements of the power source component. The corresponding sensor must be able to retain its physical characteristics under the environment with corrosive gases at high speed, temperature up to $1600^{\circ} \mathrm{C}$ in [1]. At present, the commonly used temperature sensors are mainly thermocouples, infrared sensors and optical fiber sensors and so on. Among them, the thermocouple temperature measurement range of up to $2300{ }^{\circ} \mathrm{C}$, but it needs to join the compensation circuit for control in [2]. Infrared thermometer is a non-contact measurement of the surface temperature of the object, but it is easily affected by the surface process and the environmental temperature. Optical fiber sensor used to measure temperature by optical properties of light, with anti electromagnetic interference and corrosion resistance, but the light is vulnerable to the influence of the molecular density of ambient gas in [3]. Therefore, it needs to seek new materials and sensing methods. In recent years, polymer derived ceramic (PDC) has been considered for the application of high temperature sensors. PDC is a new kind of polymer, which has good thermal stability, oxidation resistance, corrosion resistance and creep resistance. The study has found that the dielectric constant of the polymer silicon carbonitride (SiCN) produced ceramics varies with temperature, using a vector network analyzer, taking dielectric constant as intermediary, can establish the function relationship of SiCN temperature and resonance frequency in [4]. Based on the above principles, This paper designed a SiCN sensor test system, to send and receive wireless signals passive temperature sensors and real-time temperature observations.

\section{SICN SENSOR INTRODUCTION}

SiCN sensor is made based on the principle of resonant cavity, belonging to the TM010 type microwave dielectric resonator, the appearance of the sensor is cylindrical, and the top black area is the sensor antenna for receiving and reflecting microwave signals in [5], as shown in Figure 1.

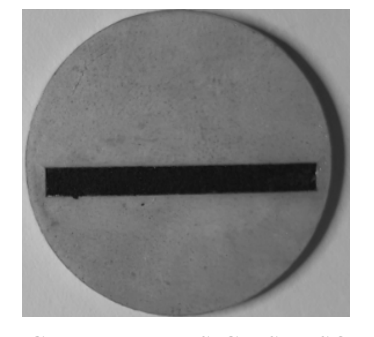

FIGURE I. THE SICN SENSOR

The physical characterization at high temperature is not only the change of the dielectric parameters of the coating material, but also the corresponding changes in the reflection signal of the broadband microwave excitation signal. According to the literature, the relationship between the dielectric constant and the resonant frequency of the TM010 resonant cavity is in [6] as follows:

$$
f_{r}=\frac{1}{2 \pi \sqrt{\mu_{0} \varepsilon_{0} \varepsilon_{r}}} \frac{\chi_{01}}{\frac{d}{2}}
$$

Among (1), $f_{r}$ is the resonant frequency. $\mu_{0}$ and $\varepsilon_{0}$ are the vacuum permeability and permittivity. the $d$ is the resonant cavity diameter. $\chi_{01}$ which is the root of the first 0 order Bessel function, and the $\varepsilon_{r}$ is the dielectric constant of the resonant cavity.And the dielectric constant with temperature change can be represented by the temperature coefficient of dielectric constant, as follows:

$$
\alpha_{\varepsilon}=\frac{1}{\varepsilon} \cdot \frac{d \varepsilon}{d t}
$$


Among (2), $\alpha_{\varepsilon}$ is the dielectric constant temperature coefficient of the temperature $t$. $\varepsilon$ is the dielectric constant of the temperature $t$. In a small temperature range. $\varepsilon$ and $t$ can be regarded as a straight line, then (2) can be transformed to the formula:

$$
\alpha_{\varepsilon}=\frac{1}{\varepsilon_{1}} \cdot \frac{\varepsilon_{r}-\varepsilon_{1}}{t_{r}-t_{1}}
$$

Among (3), $\varepsilon_{1}$ is the dielectric constant of $t_{1}$ temperature, and the $\varepsilon_{r}$ is the dielectric constant of $t_{r}$ temperature. By (1) and (3) the formula can be derived:

$$
f_{r}=\frac{1}{2 \pi \sqrt{\mu_{0} \varepsilon_{0} \cdot\left(\alpha_{\varepsilon} \varepsilon_{1} \cdot\left(t_{r}-t_{1}\right)+\varepsilon_{1}\right)}} \frac{\chi_{01}}{\frac{d}{2}}
$$

In addition to the temperature change as the variable, other parameters are constant in (4). It indicates there is a negative correlation between the temperature and the resonant frequency of SiCN sensor, that is, the temperature becomes higher while the corresponding resonant frequency becomes smaller.In this study, the main performance indicators SiCN sensor used are shown in Table 1 as below. This article only tests the sensor $\mathrm{S} 11$ in a temperature range of $25{ }^{\circ} \mathrm{C}$ to $300{ }^{\circ} \mathrm{C}$.

\section{TABLE I. SENSOR PARAMETER}

\begin{tabular}{|c|c|c|}
\hline Index & Maximum Temperature & Resonant Frequency $\left(25^{\circ} \mathrm{C}\right)$ \\
\hline Parameter & $300^{\circ} \mathrm{C}$ & $5.451 \mathrm{GHz}$ \\
\hline
\end{tabular}

By using a spectrum analyzer to test the sensor, we can get the parameters to know that when the temperature changes from 25 to 300, the frequency range of the sensor is about $27 \mathrm{MHz}$. According to the principle of the sensor, the system design requirements are analyzed:

(a) Design a (5.3 5.6) GHz frequency sweepping signal source.

(b) Establish signal transmitting and receiving channel.

(c) Acquit the feedback signal energy value

(d) Process the data and display generation.

\section{Design OF Signal TRANSMitTing AND RECEIVING SYSTEM}

According to the performance of the sensor and demand analysis, the paper needs to design a high frequency sweep frequency signal source as excitation source of SiCN sensor, and obtain the sensor return signal by the microwave devices. Through the software calculation, find the resonant frequency, convert to temperature. And observe the real-time temperature ultimately.

\section{A. System Scheme and Framework}

The system is designed as shown in Figure 2, using FPGA as the main controller, control signal source to achieve different frequency sweepping, and amplify the sweepping signal through RF gain amplifier. Then the signal into the circulator by port 1 , and output from port 2 to coaxial waveguide converter. Thereafter, the waveguide will emit the sweepping signal to SiCN sensor antenna, where the waveguide will achieve a switching signal transmission cable channels and radio channels. Sweep signal via the antenna into the SiCN sensor inside. When the frequency band of a resonant frequency coincides with the sensor, the sensor will produce resonance, and then return the feedback signal through the waveguide and output by the port 3 of the circulator. Then the coaxial detector detecte the envelope of feedback signal, at this time the output signal is low frequency, and by a series of filters and amplifiers, the FPGA control ADC module to sample and convert. Finally the results will be displayed on PC.

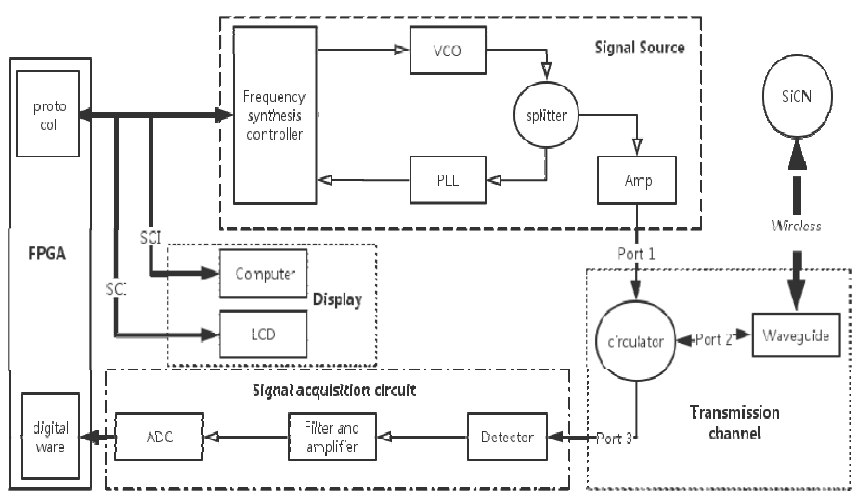

FIGURE II. SYSTEM FRAMEWORK

\section{B. Key Devices Selection and Design}

In the above scheme, the key of the system design is signal source design. In this paper, the resonance frequency of the SiCN sensor used at room temperature $\left(25^{\circ} \mathrm{C}\right)$ is about $5.5 \mathrm{GHz}$, so the range of the signal source selection must be covered by 5.5GHz. This paper selects HMC833 chip of ADI company.HMC833 is a low noise, wide, integrated phaselocked loop (PLL) of the voltage-controlled oscillator (VCO), and through the internal delta sigma modulator, it can achieve fractional. Its frequency range is $25 \mathrm{MHz} 6000 \mathrm{MHz}$, and the resolution is up to $3 \mathrm{~Hz}$, which can fully meet the measurement range of the sensor.

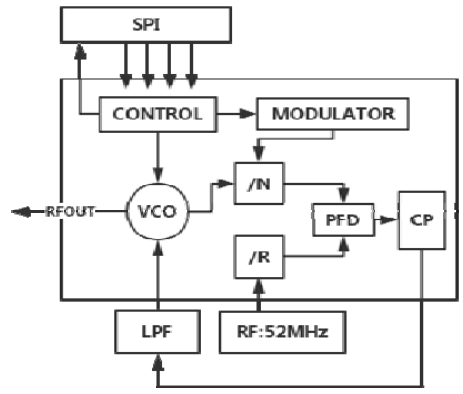

FIGURE III. HMC833 FRAMEWORK 
As shown in Figure 3, the changing of on-chip register configuration of HMC833 can be realized by SPI communication and the output frequency of the VCO is compared with an external reference frequency of the phase detector. Charge pump output error after the formation of DC signal without AC pulse, which is the ability to suppress the phase noise. DC signal through the loop filter transform to a DC voltage signal to control the VCO tuning port, so to achieve frequency lock and fractional functions.

Due to the output power of HMC833 is decreased less than $-6 \mathrm{dBm}$ in the range of $5000 \mathrm{MHz} 6000 \mathrm{MHz}$. Taking into account the rear end of the circulator, waveguides, detectors and SMA connector insertion loss(IL):

$$
\begin{aligned}
& I L_{\max }=I L_{S M A}+I L_{\text {circulator }}+I L_{\text {waveguide }}+I L_{\text {detector }} \\
& =5 * 0.2 d B+0.5 d B+0.3 d B+0.2 d B=2 d B
\end{aligned}
$$

The return loss of the circulator is about $3 \mathrm{~dB}$. The output voltage of the detector is $200 \mathrm{mV}$ when the input power is $2 \mathrm{dBm}$. Therefore, the signal source output power should meet the following conditions in order to depress the noise:

$$
\text { Pout }-(-2 d B m)=2 d B+3 d B
$$

That is, the output power should be up to at least $3 \mathrm{dBm}$. So the signal source output needs to go through the gain amplifier to improve the output power. This paper adopts the ADI company's gain amplifier HMC311 to achieve gain amplification in the cascade way.

At this point, the key components of the test system has been set up to complete and achieve the basic functions. The hardware system is shown in Figure 4.

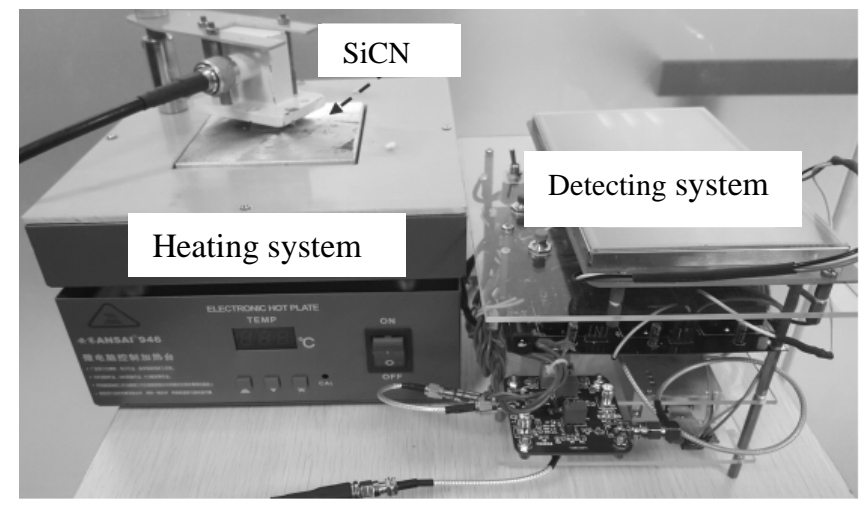

FIGURE IV. DETECTING SYSTEM AND HEATING SYSTEM

\section{TESt REsUlts ANALysis}

The heating system used in this study is the microcomputer control heating table, and the heating range of room temperature is up to $300^{\circ} \mathrm{C}$. In order to observe the relation between frequency and temperature, the paper designs a host computer by LabVIEW. The following datas are measured by the host computer and remapped through Excel.The results of the test are mainly on the waveform of the resonant frequency of the fixed temperature and the changing temperature.

\section{A. S11 Waveform at Fixed Temperature}

The experiment takes 5 fixed temperature points for S11 observation shown in Table 2 and Figure 5, where the abscissa is the value of the resonant frequency, and the ordinate value S11. The different temperatures corresponds to different resonant frequencies.

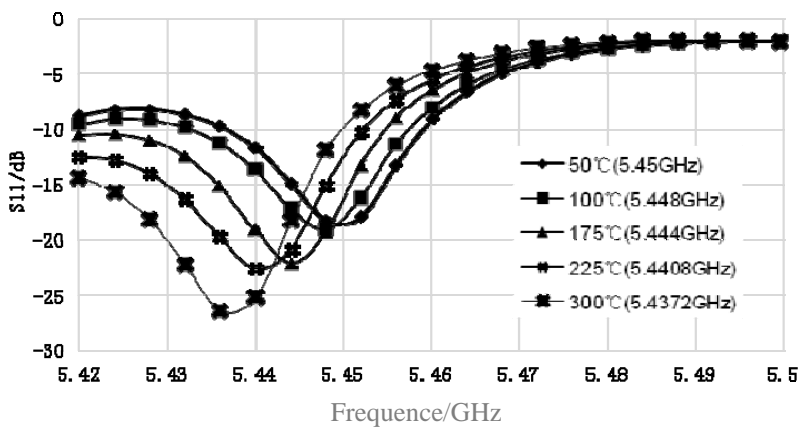

FIGURE V. S11 IN FIXED TEMPERATURE

\section{B. Resonant Frequency Waveform}

Figure 6 shows that the horizontal coordinate indicates the temperature while the longitudinal coordinate indicates the resonant frequency value, wherein the temperature is continuously variable from $25{ }^{\circ} \mathrm{C}$ to $300{ }^{\circ} \mathrm{C}$, and mark 12 temperature points. From the figure can be seen there is a negative correlation between the resonant frequency and temperature. When the temperature increases, resonant frequency decreased, and vice versa. So the temperature of the SiCN sensor can be observed, and the frequency temperature sensitivity is $45.92 \mathrm{kHz} /{ }^{\circ} \mathrm{C}$.

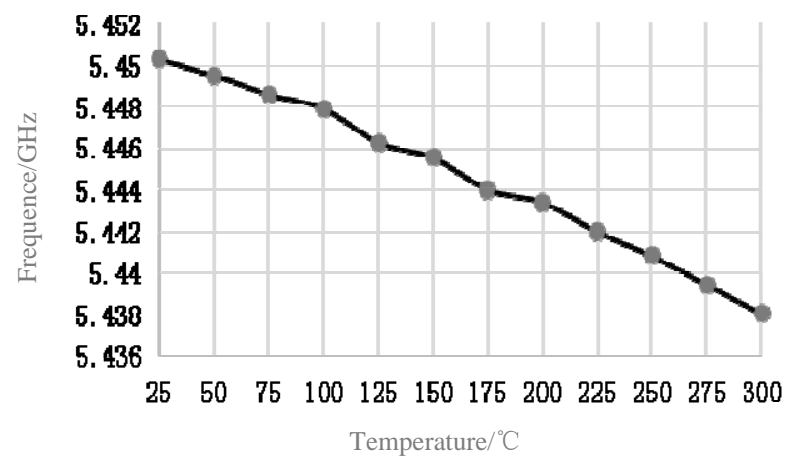

FIGURE VI. CURVE OF FREQUENCY AND TEMPERATURE

\section{CONCLUSION}

In short, the experiments prove that the system in a certain extent can replace the vector network analyzer to test relevant parameters of the SiCN temperature sensor. In contrast, the system has a better mobility, high integration and compatibility, but accuracy is needed constantly improving. At present, the 
system prototype has been completed, and the frequency temperature sensitivity is $45.92 \mathrm{kHz} /{ }^{\circ} \mathrm{C}$.

\section{REFERENCES}

[1] Frohmüller R, Beutner M, Köchig M, et al. High speed temperature measurement in gear hobbing: Part I-design, concept and physical operation mode of the infrared-camera:Part II-calibration and application of the infrared-camera-test setup and measurement results. Production Engineering, 2014, 8(1-2):73-79.

[2] .J. A. Russer et al., "A Nanostructured Long-Wave Infrared Range Thermocouple Detector," in IEEE Transactions on Terahertz Science and Technology, vol. 5, no. 3, pp. 335-343, May 2015.

[3] Zhenyang Ding, Tiegen Liu, Kun Liu, Yang Du and Dingjie Li, "Monitoring optical fiber sensor networks by optical frequency-domain reflectometry," Photonics Global Conference (PGC), 2012, Singapore, 2012, pp. 1-3.

[4] Haitao Cheng, Xinhua Ren, Ebadi, S.,Yaohan Chen,Linan An,Xun Gong.Wireless Passive Temperature Sensors Using Integrated Cylindrical Resonator/Antenna for Harsh-Environment Applications. Sensors Journal, IEEE,1453 - 1462 Volume: 15, Issue: 3, March 2015.

[5] L. Liew ,Y. Liu , R. Luo, T. Cross , L. An ,V. M. Bright, et al. Fabrication of SiCN MEMS by photopolymerizationof pre-ceramic polymer. Sens. Actuators A,Phys.,vol. 95,no. 2\&ndash;3,pp.120 $134,2002$.

[6] Sternel D C, Schäfer M, Heck M, et al. Efficiency and accuracy of fluidstructure interaction simulations using an implicit partitioned approach. Environmental Management, 1995, 19(5):703-717. 